\title{
Comment
}

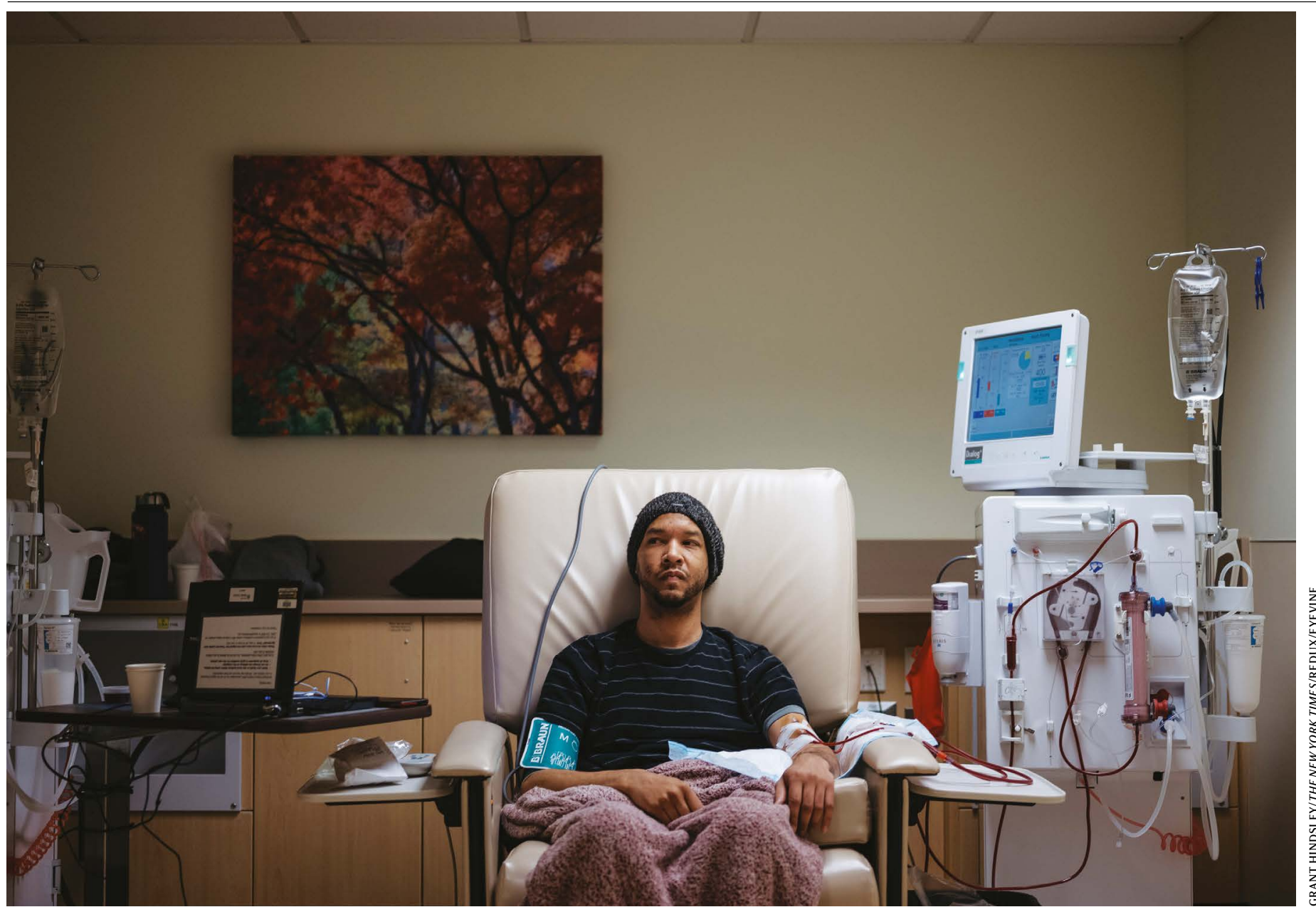

A person receiving dialysis. COVID-19 can leave people with lasting damage to their kidneys, among many other organs.

\section{Count the cost of disability caused by COVID-19}

Andrew Briggs \& Anna Vassall

\section{Focusing only on cases and} deaths hides the pandemic's lasting health burden on people, societies and economies.

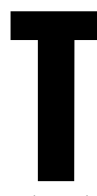

he COVID-19 pandemic is well into its second year, but countries are only beginning to grapple with the lasting health crisis. In March, a UK consortium reported that 1 in 5 people who were hospitalized with the disease had a new disability after discharge ${ }^{1}$. A large US study found similar effects for both hospitalized and non-hospitalized people ${ }^{2}$. Among adults who were not hospitalized, 1 in 10 have ongoing symptoms 12 weeks after a positive test $^{3}$. Treatment services for the long-term consequences of COVID-19 are already having to be absorbed into health and care systems urgently. Tackling this requires a much clearer picture of the burden of the disease than currently exists.

Tracking disease cases and deaths has advantages in a health emergency - they are easily collated, and, to some extent, trends can be compared across countries. But continuing the use of such simplified metrics heightens the risks of underestimating the true health impact on a population. It focuses policy and 
public discourse on the immediate prevention of deaths and on the economic impact of lockdown policies, ignoring the long-term disease-related disabilities that will also affect well-being and productivity.

To tackle the pandemic's inequitable impact, researchers must also count how COVID-19 contributes to ill health, and do so comprehensively. If scarce resources are allocated with only the death count in mind, low- and middle-income countries (LMICs) with younger populations might not receive their fair share. This could be devastating for countries that rely on the productivity of people of working age for economic development. Choosing the right metrics can also help to identify and address inequity within countries. Evidence from many nations suggest that members of minority ethnic communities are more likely to catch COVID-19 and die from it, as well as being more likely to die at a younger age ${ }^{4}$ (see also Nature 592, 674-680;2021).

Fortunately, metrics of illness exist. They inform much health policy - from cancer screening and treatment to attempts to eradicate tuberculosis. Called DALYs and QALYs (disability-adjusted life years and quality-adjusted life years), these measures capture the impact of ill health on a person's life course - combining the years of life lost because of premature death, and the years lived while experiencing the disabling consequences of disease.

As health economists, we use these metrics to understand the global burden of disease. They help us to compare the effects of prevention, treatment and social action (such as education or housing initiatives) on tackling the HIV pandemic, for example. In a recent study, we used these metrics to estimate that a death from COVID-19 results in around 5 QALYs lost, on average. We have previously used similar estimates to inform COVID-19 vaccine policy in the United Kingdom ${ }^{6}$.

Here we offer a very rough first estimate, based on simple assumptions, that as much as $30 \%$ of the COVID-19 health burden could be due to COVID-induced disability, not death. Much more needs to be done to improve such estimates so they can be acted on effectively. To design the right policies now, and invest well to deal with COVID-19 (and other pandemics) in the future, we need to use metrics that encapsulate all the consequences of a disease.

\section{Long view}

Health comprises our social, mental and physical well-being. DALYs and QALYs are already used by global institutions and

\section{COVID CASTS A LONG SHADOW}

Conditions such as heart disease gradually decrease a person's quality of life (blue) and increase their disability burden (pale orange) over their lifetime. Catching COVID-19 adds an immediate disability burden (dark orange). The disease can have a wide range of outcomes; three illustrative scenarios are shown (dashed lines).

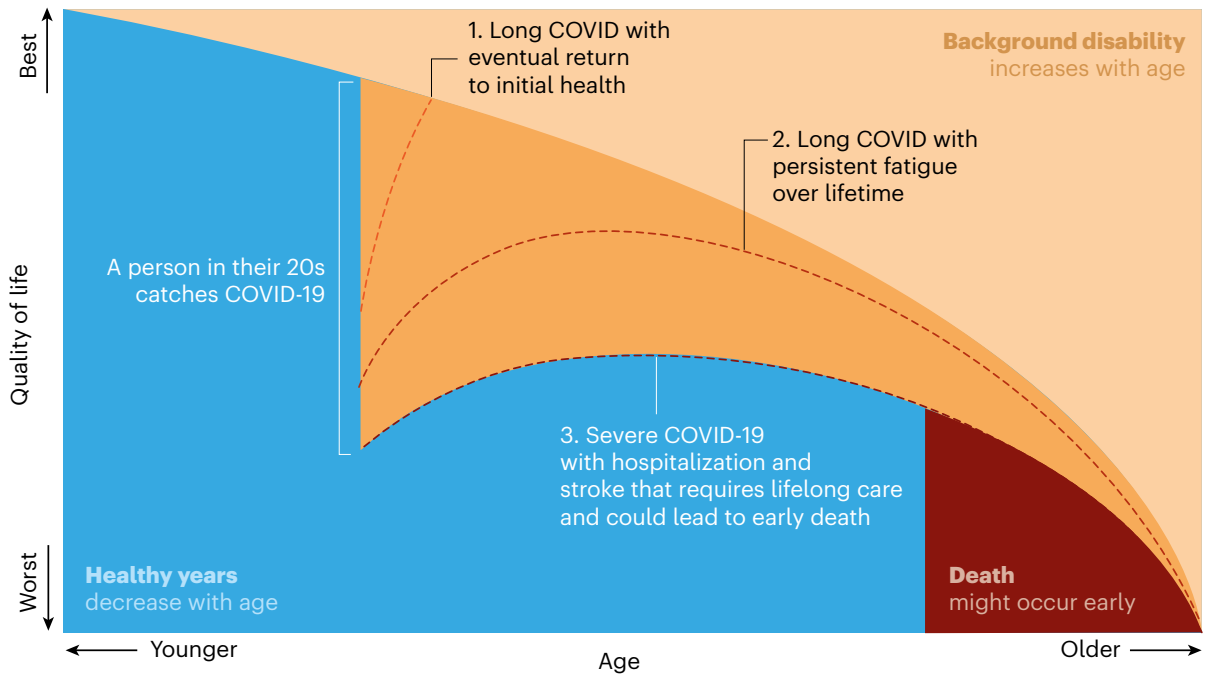

national governments to capture this holistic understanding of the burden of other diseases globally.

One DALY represents one year of healthy life lost because of illness, disability or early death. This metric has been developed and

\section{"Improved measures of disease burden can help to map the impact of COVID-19 on vulnerable communities."}

used widely by the World Bank, the World Health Organization (WHO) and the University of Washington's Institute for Health Metrics and Evaluation (IHME) in Seattle. The latter produces annual estimates of the global burden of disease (see, for example, ref. 7). DALYs are often used by governments in LMICs to set priorities across their health sectors. A 2020 estimate by Pakistan's health-services ministry found that the cost of a skilled attendant who can manage labour and delivery in low-risk pregnant women is just US $\$ 2$ for each DALY averted, for example ${ }^{8}$.

One QALY equates to one year of perfect health. This measure is mainly used by high-income countries (HICs), including the United States. That said, the US Affordable Care Act of 2010 outlawed the use of QALYs following lobbying 9 . In many countries, the metric is often used to assess the value of new technologies, such as population screening tests or innovative cell and gene therapies.
Each measure is calculated by considering the overall impact of different types of ill health - such as cancer or diabetes - during the course of a life. Although the detailed methods of calculating QALYs and DALYs differ, they can be considered functionally equivalent.

For instance, a person with a fractured limb might recover quickly if they are in their twenties. The same accident could leave a 70-yearold with reduced mobility for the rest of their life. More QALYs are lost and DALYs incurred in the second case. Likewise, the burden of HIV infection can be vastly reduced if effective drugs allow a person with the virus to live a full life.

The wide range of COVID-19 outcomes can lead to markedly different profiles (see 'COVID casts a long shadow'). A mild case of the disease in an otherwise well person might lead to 'long COVID' - a sharp decrease in overall health that can resolve after months or last for more than a year. Some people with COVID-19 experience strokes or become diabetic. Weeks of treatment in intensive care can mean that a person never returns to their former health because of damage to the brain, heart or lungs, for example. More needs to be understood about all of these scenarios so that researchers can calculate disease burdens with confidence.

\section{COVID burdens}

To illustrate how QALYs and DALYs can provide a more complete picture of COVID-19, we attempted a first rough estimate of the burden of the disease in two countries that have different demographics: Pakistan and the United 
Kingdom (see 'Life and health lost'). The population of Pakistan, in common with many LMICs, has a high proportion of younger age groups; the United Kingdom, like other HICs, does not. It should be noted that our sketch is illustrative only, because we extrapolated from UK data on the rates of hospitalized people needing long-term care. The two nations have differing health systems, pre-existing patterns of co-morbidity and so on; and many people who are not hospitalized with COVID-19 also experience lasting effects.

We approximate that the health burden due to COVID-induced disability across all age groups could be around $30 \%$ overall. We arrived at this crude figure using modest assumptions about the duration and severity of COVID-19-related disease (see Supplementary information). This number also assumes a loss of 0.5 DALYs for an average case of long COVID, regardless of age, and includes DALYs lost as a result of COVID-19 deaths, using standard methods ${ }^{5}$.

This exercise hints at how much of the longterm health impact we could be missing by counting only deaths.

We can also compare the DALYs lost across age groups. The demographics in Pakistan could place more of the estimated burden of sickness on people of working age. More research is needed to assess whether this is the case: death statistics, in particular, could be under-reported among older people in many countries.

In the United Kingdom, too, counting COVID disabilities gives a different picture of the impact of the pandemic. The steep increase in death rate with age means that deaths dominate the DALYs lost for the very old. But the share of cases is much flatter across the adult population. Younger people are much more likely to be left sick than dead by COVID-19. Metrics that consider a person's stage of life and disease could fundamentally shift both public understanding and the policy response to COVID-19.

\section{Metrics for policy}

QALYs and DALYs enable governments to balance resources between lockdowns, quarantine, social distancing, ventilation, masking, vaccination, testing, treatment and long-term care for COVID-19. It is crucial to get these trade-offs right in LMICs such as Pakistan, where the public purse has only US $\$ 15$ per person to spend on health, according to World Bank data. The price of COVID-19 vaccination is considerable. The cost of administering two doses of COVID-19 vaccine in Pakistan is estimated to be $\$ 3.15$ per person, without factoring in the cost of the doses themselves ${ }^{10}$.

As the market for COVID-19 vaccines and therapeutics becomes more crowded, using a common metric for disease burden and value

\section{LIFE AND HEALTH LOST}

Metrics that capture the overall burden of ill health, rather than simply counting deaths, hint at how COVID-19 might affect populations in Pakistan and the United Kingdom in the long term.

\section{Different demographics}

Pakistan's population is predominantly young; the United Kingdom's is more evenly distributed across age groups.

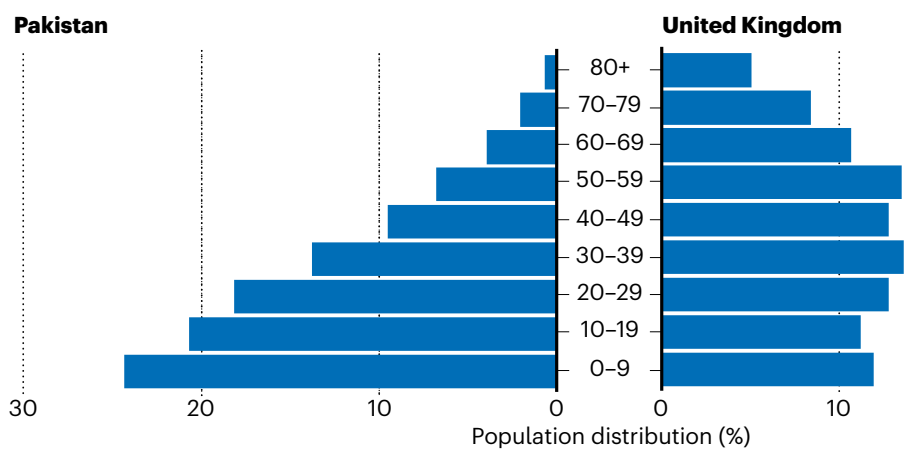

20

\section{DALYs lost to COVID-19}

Estimates using disability-adjusted life years (DALYs) suggest that, in Pakistan, most of the health burden of COVID-19 could fall on people aged 60 or under.

\section{Pakistan}

0.31 total

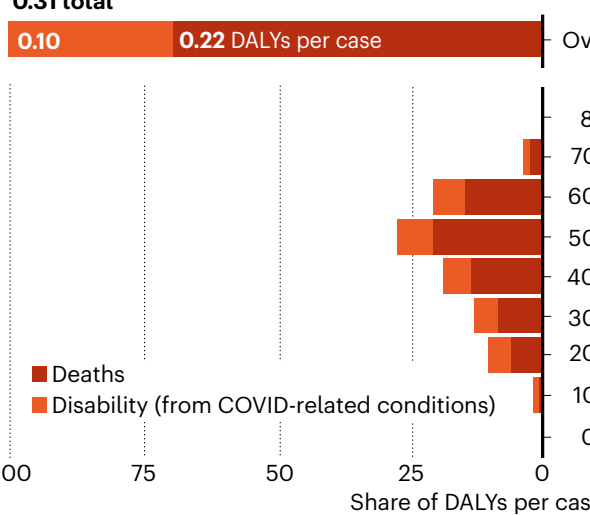

United Kingdom

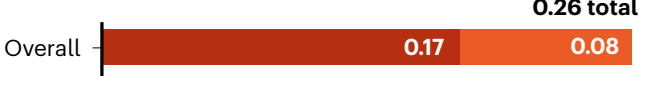

.26 total

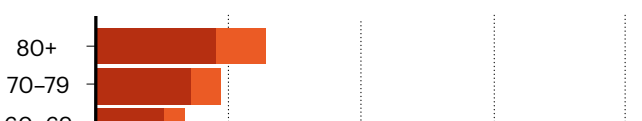

assessment will become important for HICs, too. It seems self-evident that vaccines are a wise investment for wealthy nations, given the huge toll that the pandemic places on economies. Indeed, for this reason, we think it might be cost-effective for HICs to vaccinate the entire global population, not just their own

\section{"Younger people are much more likely to be left sick than dead by COVID-19."}

citizens. But countries could have to decide, for example, whether the antibody cocktail casirivimab and imdevimab is a sensible use of heath budgets, now that data are emerging on its potential effectiveness and before a price has been set (see go.nature.com/3wh9kjy).

An estimated $6 \%$ of the global population is living with two or more conditions (co-morbidities) that put them at high risk of death or disability from COVID-19 (ref. 11). QALYs and DALYs can help governments to compare interventions targeted at such co-morbidities with those that directly address COVID-19. For instance, managing non-communicable diseases such as diabetes requires substantial increases in funding globally ${ }^{12}$. Without a clear analysis of these factors, pressure to allocate scarce resources to COVID-19 could reduce the availability of services for non-communicable disease. This might worsen overall well-being in the long run ${ }^{13}$.

Improved measures of disease burden can help to map the impact of COVID-19 on vulnerable communities. In the United States, Black and Latinx people and Indigenous Americans are roughly three times more likely to be hospitalized with the disease than are white or Asian, non-Hispanic people (Nature 592, 674-680; 2021).

Longer-term outcomes are also likely to be affected by underlying health disparities and unequal access to care and treatment. And for people without a social safety net, chronic disease can push them into poverty ${ }^{14}$.

Metrics that capture how ill health, disability, stigma and poverty interact with COVID-19 could prove pivotal in efforts to overcome the disease. Other infectious diseases, such as tuberculosis, commonly become entrenched in communities that are unable to fully protect themselves against risk, owing to social and economic barriers ${ }^{14}$. 
In the ongoing global HIV epidemic, countries that recognized and supported community action for vulnerable people ${ }^{15}$ early on were able to prevent wider transmission over time.

\section{Next steps}

It has always been difficult to assess the comprehensive burden of health and inequity. This is particularly hard in settings where disease surveillance and health-information systems are weak. The COVID-19 pandemic has shown the importance of these systems, and should drive a global effort to improve them.

Poorer nations are streets ahead of wealthy ones in their use of DALYs. The metric has highlighted emerging patterns of HIV in south India, for example, and has helped to identify the risk factors behind them ${ }^{16}$. They also inform health planning. Ethiopia ${ }^{17}$ and Pakistan both recently used DALYs to define the package of services required to roll out universal health care in their countries.

On the basis of this infrastructure, global DALYs attributable to COVID-19 are likely to appear in the next burden of disease estimates from both the WHO and the IHME. Due to be released in the second half of 2021, these should be the first comprehensive assessments of the relative health burden from COVID-19 globally. They could support the change in measurement and perception that

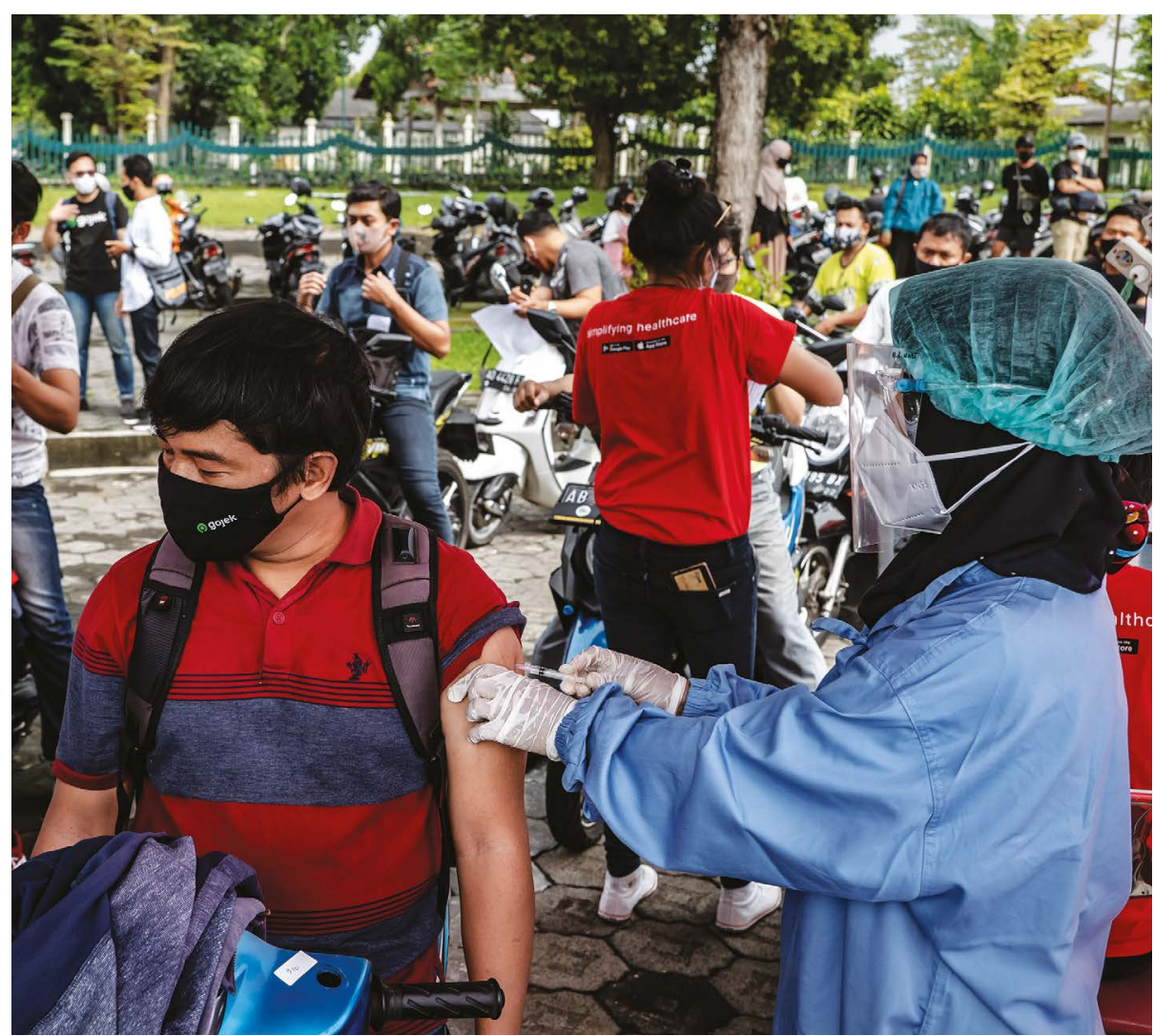

Indonesia is prioritizing vaccinations for people of working age (18-59-year-olds) to aid its economic recovery. we are highlighting, but they are likely to suffer from a paucity of data, with estimates being highly uncertain.

These data gaps appear because many LMICs lack the reporting infrastructure required to calculate QALYs and DALYs. Apart from South Africa, most countries in sub-Saharan Africa have been unable to count excess deaths from COVID-19, for example, because of inadequate registration systems. Scaling up of testing remains one of the most urgent public-health needs. But it is challenging for governments to prioritize the gathering of information when they cannot afford essential medicines ${ }^{18}$. The scientific community and HICs should ramp up investment in health information systems to capture service use, morbidity and mortality.

Research funding to build a global picture of COVID-19-related disease is also needed. The full range of symptoms and consequences is not yet known. These might also depend on a person's underlying health status and access to care. Longitudinal studies, simKingdom ${ }^{1}$ and other HICs ${ }^{19,20}$, should be initiated globally. These should assess the mental-health effects and long-term economic impacts of living with chronic diseases in the wake of COVID-19. Countries such as South Africa - which developed infrastructure to track populations over time for epidemics ilar to those beginning to report in the United such as HIV - should lead the way.

Data on both deaths and disease in vulnerable groups is often the most challenging to collect, particularly where access to services is poor. But it can be done. Kenya, for instance, is already conducting surveys that can potentially feed into real-time models of COVID-19 transmission and impact ${ }^{21}$. These should be linked with burden-of-disease estimates.

As we count the devastating losses from COVID-19- of loved ones, jobs, communities, security - the lasting loss of health must also be tallied. Without the right metrics, we can see, understand and respond to only a fraction of the problem.

\section{The authors}

Andrew Briggs is professor of health economics in the Department of Health Services Research \& Policy, London School of Hygiene \& Tropical Medicine, UK. Anna Vassall is professor of health economics in the Department of Global Health \& Development, London School of Hygiene \& Tropical Medicine, UK; and chair in global health at the Amsterdam Institute of Global Health and Development, University of Amsterdam, the Netherlands.

e-mails: andrew.briggs@lshtm.ac.uk; anna.vassall@lshtm.ac.uk

Supplementary information accompanies this article: see go.nature.com/3fanp9f.

1. PHOSP-COVID Collaborative Group. Preprint at medRxiv https://doi.org/10.1101/2021.03.22.21254057 (2021).

2. Al-Aly, Z., Xie, Y. \& Bowe, B. Nature https://www.nature. com/articles/s41586-021-03553-9 (2021).

3. National Institute for Health Research. Living with COVID19: Second Review https://doi.org/10.3310/ themedreview_45225 (NIHR, 2021).

4. Public Health England. Disparities in the Risk and Outcomes of COVID-19 (PHE, 2020).

5. Briggs, A. H. et al. Health Econ. 30, 699-707 (2021).

6. Sandmann, F. G. et al. Lancet Infect. Dis. https://doi. org/10.1016/S1473-3099(21)00079-7 (2021).

7. Vos, T. et al. Lancet 396, 1204-1222 (2020).

8. Ministry of National Health Services, Regulations and Coordination. Universal Health Coverage Benefit Package of Pakistan (Government of Pakistan, 2020); available at https://go.nature.com/3upgx8t

9. Neumann, P. J. \& Weinstein, M. C. N. Engl. J. Med. 363 1495-1497 (2010).

10. Griffiths, U. et al. Costs of Delivering COVID-19 Vaccine in 92 AMC Countries: Updated Estimates from COVAX Working Group on Delivery Costs (WHO, Gavi \& UNICEF, 2021).

11. Clark, A. et al. Lancet Glob. Health 8, e1003-e1017 (2020).

12. Nugent, R. Glob. Heart 11, 371-374 (2016)

13. Abbas, K. et al. Lancet Glob. Health 8, e1264-e1272 (2020). 14. Puteh, S. E. W. \& Almualm, Y. Health Syst. Policy Res. 4, 1 (2017).

15. Medley, G. F. \& Vassall, A. Science 357, 156-158 (2017). 16. Vassall, A. et al. Lancet Glob. Health 2, e531-e540 (2014). 17. Verguet, S. et al. Nature Med. 27, 380-387 (2021).

18. Watkins, D. A. et al. Lancet Glob. Health 8, e829-e839 (2020).

19. Walle-Hansen, M. M., Ranhoff, A. H., Mellingsæter, M., Wang-Hansen, M. S. \& Myrstad, M. BMC Geriatr. 21, 199 (2021).

20. Daugherty, S. E. \& Guo, Y. Br. Med. J. 373, n1098 (2021)

21. Quaife, M. et al. BMC Med. 18, 316 (2020). 